\title{
TCAS/ ADS-B Integrated Surveillance and Collision Avoidance System
}

\author{
Yajun $\mathrm{Xu}$ \\ Aviation Engineering Institute \\ Civil Aviation Flight University of China \\ Guanghan Sichuan,China \\ genius98@126.com
}

\begin{abstract}
The air collision avoidance system is an important measure to ensure the flight safety. One of the sticking point of collision avoidance is surveillance precisely and reliably. So, it is necessary to develop an independent and reliable air-air surveillance system. A TCAS/ ADS-B integrated surveillance and collision avoidance system was proposed in this paper. This system is based on the TCAS original collision avoidance function, and integrated with ADS-B broadcasting information .Using the present statistical model and data fusion algorithm, the integrated system can get optimal fusion track estimate. The simulation results show that this integrated system can improve TCAS tracking accuracy, improve the surveillance precision and collision avoidance reliability.
\end{abstract} fusion

Keywords-TCAS; ADS-B; Integrated surveillance; Data

\section{INTRODUCTION}

The TCAS and the ATC ground Surveillance both interrogate the transponder on the frequency of $1030 \mathrm{MHz}$, and get the response signals on the frequency of 1090 $\mathrm{MHz}[1][2][3]$. In order to reduce the radio interruption, it is necessary to reduce the interrogating rate of TCAS, thus, TCAS can not estimate the target's position in real time, so the position error is a sixty-four-dollar question for TCAS tracking. The ability of conflict predicting and collision avoidance of TCAS becomes complex with the increasing of traffic flow.

Automatic Dependent Surveillance- Broadcast (ADS-B) is an application which allows the aircraft to broadcast its state vector (horizontal and vertical position, horizontal and vertical velocity) and other information to neighboring aircraft and nearby ground station periodically. So, The ADS-B can provide better situation awareness of local air space to all parties concerned. The applications for ADS-B include ATC display of traffic, runway incursion detection and alerting, and Cockpit Display of Traffic Information (CDTI)[4][5] [6] [7].

ADS-B is known as dependent and cooperative surveillance. Dependent because it relies on the availability of onboard navigation and transmission equipment, and cooperative because information can only be obtained from properly equipped aircraft.

For all the causes described above, the Federal Aviation Administration (FAA) and the National Aeronautics and Space Administration (NASA) are investigating the suitability of ADS-B technology to support the TCAS system, i.e. TCAS/ADS-B integrated surveillance system.

\section{A. TCAS Collision Avoidance Principle}

- The Traffic Alert and Collision Avoidance System (TCAS) transmits interrogation signals to other ATC transponder equipped airplanes, and receives response signals from other ATC transponder equipped airplanes, to get altitude, range, and bearing data of other ATC transponder equipped airplanes. The aircraft with TCAS is called own aircraft, and the aircraft with transponder around it is called a target.

- Using the information from the response signals and altitude of own airplane, TCAS computer calculates the relative movement between own airplane and the target, then calculates how close the target will be to own airplane at the closest point of approach (CPA).According to the time until the target will be at CPA to the TCAS airplane, the TCAS forms two protected areas around its own airplane, the Traffic Advisory (TA) area and Resolution Advisory( RA) area. The TA time varies within 20 48s, the RA time varies within 15 35s[1][2][3], as shown in figure 1.If the target penetrates either the RA protected areas ,or the RA protected area, the TCAS will provide position indicating on the CDTI .If the target penetrates the RA area, the TCAS will provide aural and visual resolution advisory to avoid collision[1][2].

\section{B. Comparison between TCAS and ADS-B}

- The TCAS is a collision avoidance system, In order to estimate whether the target aircraft enter into the TA or RA protected area or not, The TCAS must interrogate the ATC transponder and receive the response signal. The ADS-B broadcasts its information automatically without interrogation.

- The TCAS calculates the conflict only based on the present and history position and velocity, the ability of conflict predicting becomes complex with the increasing of traffic flow. The ADS-B get the position and velocity from other aircraft directly, so it's easy to calculate the track trend of other aircraft .

- The TCAS interrogates the ATC transponder on the frequency of $1030 \mathrm{MHz}$, and receives the ATC transponder replying on the frequency of $1090 \mathrm{MHz}$. The ADS-B broadcasts its information also on the frequency of $1090 \mathrm{MHz}$. So, the TCAS can receive 
the ATC transponder's replying signal, as well as the ADS-B broadcasting signal.

\section{TCAS/ADS-B INTEGRATED SURVEILLANCE SYSTEM}

In the TCAS/ADS-B integrated surveillance system, the TCAS can get the target's position and velocity information directly broadcasted from the ADS-B without interrogating the transponder. So it can reduce the TCAS interrogating rate, reduce the radio interruption, maintain the independence of collision avoidance of TCAS, and increase the surveillance precision.

The maximum horizontal front surveillance range of TCAS is $40 \mathrm{~nm}$, the aft surveillance area and cross side surveillance area are less [1] , as shown in figure 1.The surveillance area of ADS-B is no less than $100 \mathrm{~nm}$ in all directions [6], as shown in figure 1.

The surveillance area of TCAS/ADS-B integrated surveillance system can be divided into the ADS-B only surveillance area and the TCAS / ADS-B integrated surveillance area.

a. The ADS-B only surveillance area is within the ADSB surveillance area and beyond the TCAS surveillance area , as shown in figure 1.

b. The TCAS /ADS-B integrated surveillance area is within the TCAS original surveillance area, as shown in figure 1.

TCAS/ADS-B integrated surveillance area can be divided into low frequency interrogating area and normal frequency interrogating area ,

(a).Low frequency interrogating area is within the TCAS surveillance area and beyond the TCAS protect area, as shown in figure 1.In this area, the TCAS interrogates the ATC transponder at low frequency, e.g. once per 10s [1] [5].

(b). Normal frequency interrogating area is the TCAS TA protected area and the TCAS RA protected area , as shown in figure 1.In this area, the TCAS interrogates the ATC transponder at normal frequency, e.g. once per 1s[1] [5].

Within the ADS-B only surveillance area, the ADS-B can get the target's position and velocity information broadcasted directly from the ADS-B on the target aircrafts. Using the position and velocity information, the state and the track of the target aircraft can be evaluated.

When the target aircraft enters into the TCAS/ADS-B integrated surveillance area. The TCAS interrogates at low frequency of once per 10s first. During this stage, the TCAS data is used to verify the ADS-B information, the recent effective TCAS data and effective ADS-B data are compared, then fused, at last, the fused track can be gotten.

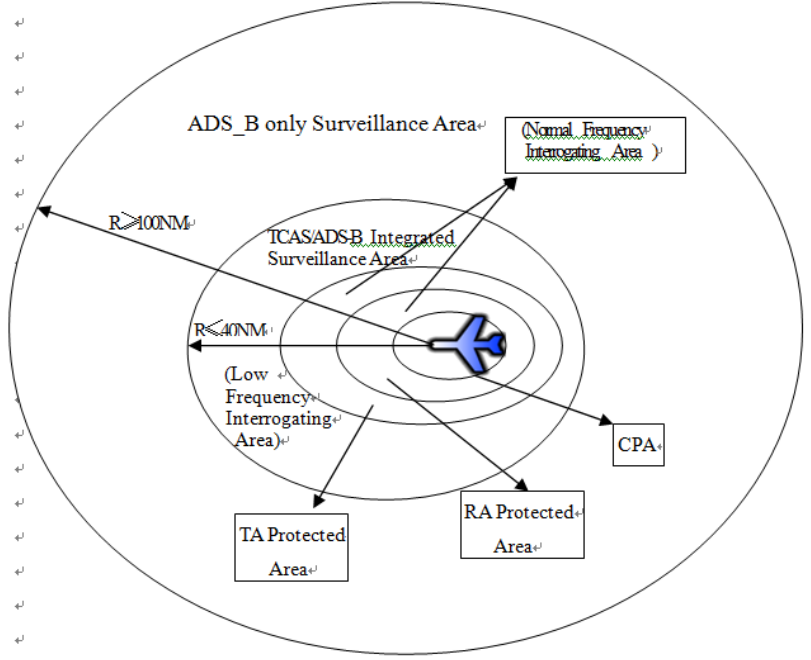

Figure 1. The TCAS/ADS-B integrated horizontal surveillance area

When the target aircraft enters into the TCAS TA protected area or the TCAS RA protected area, the TCAS restore its normal interrogating frequency of once per $1 \mathrm{~s}$. During this stage, the TCAS real-time data and the ADS-B real-time data are fused,then, the state and the position of the target aircraft are evaluated accurately. Then, the accurate position of the target aircraft is sent into the decision-making unit in the TCAS. The TCAS provides aural and visual collision avoidance resolution advisory to the flight crew.

In this type of TCAS/ADS-B integrated surveillance system, the final fused data is sent into the decision-making unit in the TCAS, the ADS-B is only used as an input interface to provide external information for the TCAS. So, the TCAS still maintains its basic collision avoidance function.

The brief track information and estimated track of the target aircraft can be displayed on the CDTI. The information displayed on the CDTI has Character of visualization. Using the information displayed on the CDTI, the flight crew can monitor the motion of the target aircraft visually , so the situation awareness and collision resolution ability of flight crew can be improved.

The executing flow of the TCAS/ADS-B integrated surveillance is shown in figure2.

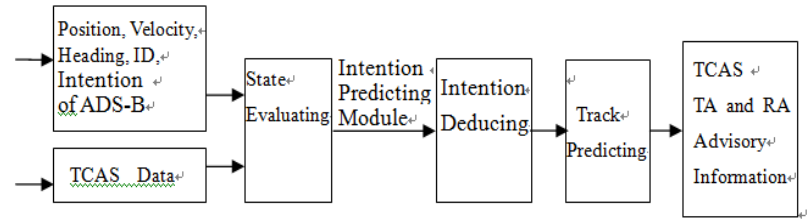

Figure 2. The TCAS/ADS-B integrated surveillance executing flow chat

\section{ALgORITHM OF DATA FUSION}

At the first stage of integrated surveillance, the interrogating frequency of TCAS is set as $1 / 10 \mathrm{~Hz}$, the broadcasting frequency of ADS-B is set as $1 \mathrm{~Hz}$.

At the second stage of integrated surveillance, the TCAS interrogating frequency and the ADS-B broadcasting 
frequency are both set as $1 \mathrm{~Hz}$. During this stage, the TCAS data and ADS-B data are preprocessed first, then, fused in real time.

The block diagram of the TCAS/ADS-B integrated surveillance data fusion system is shown as figure 3.In order to improve the state and the track estimation precision, a feedback loop is used in this system. In order to get the optimal TCAS track and optimal ADS-B track, the TCAS track and the ADS-B track are filtered in the local TCAS Kalman filter and the local ADS-B Kalman filter separately. Then, the optimal TCAS track and the optimal ADS-B track are fused at the track fusion node to get the fused track, then, the fused track is filtered in the whole Kalman filter, so, the optimal estimate track is gotten, the precision and reliability of the integrated surveillance system is improved.

Whenever the track fusion node receives a group of local tracks of TCAS and ADS-B, the fusion algorithm will extrapolate the state of the post fused track to the time of receiving the local tracks, associate and fuse it with the new local tracks, then, the state vector estimate of present integrated track is gotten, so, the present integrated track is gotten,. When a new group of local tracks is received, the above steps are repeated [8] [9].

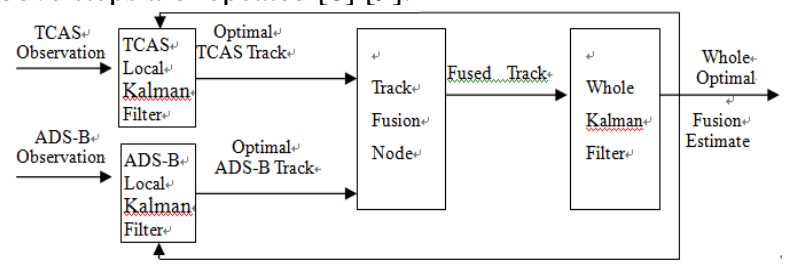

Figure 3. The block diagram of the integrated surveillance data fusion system

Considering the requirement of air space surveillance precision and the real-time, the present statistical model is selected to track the target in this paper. The equation of state is[10]

$$
X(k+1)=F(k) X(k)+G(k) u(k)+V(k)
$$

Where

$X(k)$ is the state Variable ; $F(k)$ is the transformation matrix; $G(k)$ is the input control matrix,; $u(k)$ is the known input control signal; $V(k)$ is the zero mean white Gaussian noise of $Q(k) \quad 0$.

The TCAS and ADS-B observes the target aircraft independently, Using the TCAS observation and the ADS-B observation, the two local Kalman filters estimate the optimal TCAS track and the optimal ADS-B track. The model of No.i local filter is :

$$
\begin{gathered}
X_{i}(k+1)=F(k) X_{i}(k)+G(k) u(k)+V(k) \\
z_{i}(k)=H_{i}(k) X_{i}(k)+W_{i}(k)
\end{gathered}
$$

$H(k)$ is the observation matrix; $W(k)$ is the observation noise sequence.

The TCAS and ADS-B estimates the optimal local state $X_{i}(k)$ using the local Kalman filter. For the present statistical model, Kalman filter algorithm of the No.i local filter is:

$$
\begin{gathered}
\hat{X}_{i}(k+1 / k+1)=\hat{X}_{i}(k+1 / k)+K(k+1)\left[\mathrm{z}(k+1)-H_{i}(k+1) \hat{X}_{i}(k+1 / k)\right] \\
\hat{X}_{i}(k+1 / k)=F(k) \hat{X}_{i}(k / k)+G(k) u(k) \\
\left.\mathrm{Ki}(\mathrm{k}+1)=\mathrm{Pi}(\mathrm{k}+1) / \mathrm{k}) \mathrm{Hi}^{\mathrm{T}}(\mathrm{k}+1)[\mathrm{Hi}(\mathrm{k}+1) \mathrm{Pi}(\mathrm{k}+1) / k] \mathrm{Hi}^{\mathrm{T}}(\mathrm{k}+1)+\mathrm{Ri}(\mathrm{k}+1)\right]-1
\end{gathered}
$$

$$
\mathrm{Pi}(\mathrm{k}+1) / \mathrm{k})=\mathrm{F}(\mathrm{k}) \mathrm{Pi}(\mathrm{k} / \mathrm{k}) \mathrm{F}^{\mathrm{T}}(\mathrm{k})+\mathrm{Q}(\mathrm{k})
$$

$$
\left.\left.\mathrm{P}_{\mathrm{i}}(\mathrm{k}+1) / \mathrm{k}+1\right)=\left[\mathrm{I}-\mathrm{K}_{\mathrm{i}}(\mathrm{k}+1) \mathrm{H}_{\mathrm{i}}(\mathrm{k}+1)\right] \mathrm{P}_{\mathrm{i}}(\mathrm{k}+1) / \mathrm{k}\right)
$$

Where, $K(k)$ is gain of the Kalman filter.

After processed in the side-by-side local Kalman filters, two local optimal state estimates $\hat{X}_{\text {TCAS }}(k)$ and $\left.\hat{X}_{A D S-B}(k)\right)$ are gotten. The $\hat{X}_{\text {TCAS }}(k)$ and $\hat{X}_{A D S-B}(k)$ will be fused in the whole Kalman filter.

Considering that the whole optimal state estimate is the linear combination of the two local optimal state estimates $\hat{X}_{\text {TCAS }}(k)$ and $\hat{X}_{A D S-B}(k)$ ),according to the principle of minimum variance, the two local optimal state estimates $\hat{X}_{T C A S}(k)$ and $\left.\hat{X}_{A D S-B}(k)\right)$ are fused in the whole Kalman filter by the means of weighted average. The whole optimal fusion estimate is[11]:

$$
\hat{X}=W_{1} \hat{X}_{T C A S}+W_{2} \hat{X}_{A D S-B}
$$

Where, $W_{1}$ and $W_{2}$ are the undeterminded coefficient matrix. And $W_{1}+W_{2}=I$ ( $I$ is a $n \times n$ unit matrix). $W_{1}$ and $W_{2}$ make $\hat{X}$ a no deviation least variance estimation, i.e. $\hat{X}$ meets : 1)no deviation, i.e. $\mathrm{E}[(\mathrm{X}-\hat{X})]=0$; 2)the estimation error of $\hat{X}$ is the least,

i.e. $\hat{P}=E\left[(X-\hat{X})(X-\hat{X})^{T}\right)$ is the least, that is to say, the whole optimal fusion estimation variance is less than either of the local optimal estimate variance.

So, $E(X-\hat{X})=E\left(X-W_{1} \hat{X}_{1}-W_{2} \hat{X}_{2}\right)=0$. It is certificated that by the means of the weighted average matrix, the variance of the fusion estimation is less than the variance of either local estimate, i.e. $\hat{P} \leq \hat{P}_{1}, \hat{P} \leq \hat{P}_{2}$. So the follow conclusion can be drawn: The whole optimal fusion estimate is better than either of local optimization estimate.

For the $\hat{X}$ meets both condition 1)and condition 2), $\hat{X}_{T C A S}$ and $\hat{X}_{A D S-B}$ are irrelated, If the estimate error variances for The TCAS and ADS-B are $\hat{P}_{11}$ and $\hat{P}_{22}$, the estimate error covariances are $\hat{P}_{12}, \hat{P}_{21}$,

$$
\begin{aligned}
& \text { then } \quad \hat{P}_{12}=\hat{P}_{21}=0 . \\
& \text { so } \\
& \qquad \begin{array}{l}
\hat{X}=\left(P_{11}^{-1}+P_{22}^{-1}\right)^{-1}\left(P_{11}^{-1} \hat{X}_{1}+P_{22}^{-1} \hat{X}_{2}\right) \\
\hat{P}=\left(P_{11}^{-1}+P_{22}^{-1}\right)^{-1}
\end{array}
\end{aligned}
$$


By the means of weighted average of $\hat{X}_{T C A S}(k)$ and $\hat{X}_{A D S-B}(k)$, the optimal state estimate of the target is gotten

\section{VALIDATION OF THE PRESENT TECHNIQUE}

In order to validate the validity of the data fusion algorithm used in the TCAS/ADS-B surveillance system, the simulation is made as follow: The sampling period of TCAS and ADS-B are both set as 1s, the original position of the target aircraft is set as 0 , the original velocity of the target aircraft is set as: $\boldsymbol{v}_{\mathrm{xo}}=180 \mathrm{~m} / \mathrm{s}, \boldsymbol{v}_{\mathrm{y} 0}=0$; the original acceleration of the target aircraft is set as $\mathrm{a}=0$.If the target aircraft flies at constant speed of $\boldsymbol{v}_{\mathrm{xo}}=180 \mathrm{~m} / \mathrm{s}, \boldsymbol{v}_{\mathrm{y} 0}=0$ for 10s; Then flies at constant acceleration of $3 \mathrm{~m} / \mathrm{s} 2$ for $10 \mathrm{~s}$; then, flies at constant speed for 20s. The observation noise variance of the TCAS is $50 \mathrm{db}$. The observation noise variance of the ADS-B is $40 \mathrm{db}$. The two observation noise variances are both white Gaussian noise. The simulation result is shown as figure 5 .

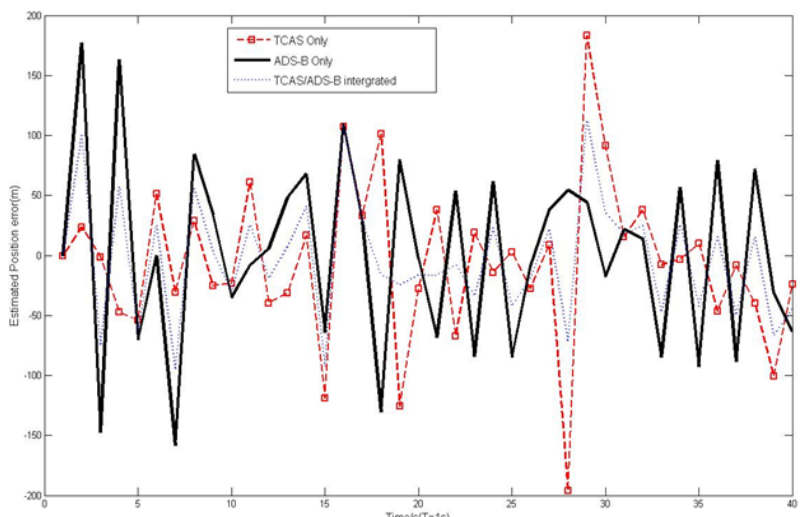

Figure 4. The integrated surveillance data fusion algorithm simulation

The simulation result shows that the maximum optimal fusion estimate is $100 \mathrm{~m}$, and the maximum optimal TCAS estimate is about $180 \mathrm{~m}$, the maximum optimal ADS-B estimate is about $200 \mathrm{~m}$.So, the integrated fusion estimate is better than either of the optimal TCAS estimate and the optimal ADS-B estimate .

\section{CONCLUSION}

The TCAS.ADS-B integrated surveillance system is a surveillance system based on the original collision avoidance function of TCAS. In this integrated surveillance system ,the TCAS listens in the ADS-B broadcasting information of the target aircraft, and fusing the TCAS data and the ADS-B data, so ,it can reduce the TCAS radio frequency interruption, improve the surveillance precision and extend the surveillance. In this integrated surveillance system, the observation of TCAS and ADS-B are fused by the means of weighted average. The simulation result shows that the optimal fusion estimate is better than either of local optimal estimate.

\section{ACKNOWLEDGMENT}

I would like to express my gratitude to all those who have helped me during the writing of this paper. I gratefully acknowledge the help of my staff member Junhui $\mathrm{Li}$ and Huazhong Zhang. I do appreciate their patience, encouragement, and professional instructions during my paper writing. Also, I would like to thank Miss Guiping He, who kindly gave me a hand when I was making validation of the present technique.

\section{REFERENCES}

[1] RTCA. "Minimum Operational Performance Standards (MOPS) for Traffic Alert and Collision Avoidance System II (TCAS II) Hybrid Surveillance,” [S]. DO-300,Issued 12-13-06,Prepared by SC-147.

[2] Daniel.Storm.Hicok,Rannoch,Corporation,Alexandria,Virginia,Derric $\mathrm{k}$ Lee. Rannoch Corporation, Alexandria,Virginia. "Application of ADS-B for airport surface surveillance," Avionics systems Conference. 1998. Proceedings. 17th DASC. The ALAA/IEEE/SAE .

[3] Zhang Jun. Modern air traffic management,[M].Beijing:Beijing University of Aeronautics and Astronautics Press.2005.pp:190-202.

[4] Ming-Shih Huang, Ram M. Narayanan. "Mutiple targets estimate and tracking for ADS-B radar system,"[J]. IEEE Digital Avionics Systems Conference,2008. DASC 2008. IEEE/AIAA 27th.

[5] Bernays,D.J.,Drumm,A.C and Shank. "Valodation technique for ADS-B surveillance data,’[J].IEEE Digital Avionics Systems Conference-Proceedings, 2002.

[6] RTCA. “ Minimum Operational Performance Standards for 1090 $\mathrm{MHz}$ Extended Squitter Automatic Dependent Surveillance Broadcast (ADS-B) and Traffic Information Services - Broadcast (TIS-B) Volume 1,”[S]. DO-260A, RTCA, Inc., Dec. 2006.

[7] Aeroflex . "XPDR/DME/TCAS/ADS-B/TIS/TIS-B TEST SET IFR 6000 Operation Manual,”[S]. 2007.

[8] Yang Wanhai. “ Multi-sensor data fusion and application,”[M].Xi'an: Xian Electronic Sience \& Technology University Press.2004. pp:150168.

[9] He You,Xiu Jianjuan. Radar data fusiom and applocation(Edition 2),[M].Beijing:Electronic Industry Press.2009. pp:108-132.

[10] Sen Gongxun, Sun Jianfeng. Information fusion theory and its application to inertial/ astronomy/GPS integrated navigation system, [M]. Beijing: National Defence Industry Press.1998. pp:121-145. 